\title{
A New Pre-Order Relation for Set Optimization using l-difference
}

\author{
Emrah Karaman
}

\begin{abstract}
A new relation on the subset of the space is defined via $\ell$-difference in this work. This is a pre-order relation on the family of nonempty sets. Some relations between this pre-order relation and well-known order relations are investigated. Also, the solution points of a set-valued optimization problem via set and vector approaches are examined.
\end{abstract}

Keywords: Pre-order relation, Set optimization, Vector approach

2010 AMS: Primary 80M50, Secondary 90C26

Karabük University, Faculty of Science, Department of Mathematics, 78050 Karabük, Turkey, ORCID: 0000-0002-0466-3827

Received: 24 June 2021, Accepted: 24 August 2021, Available online: 11 October 2021

\section{Introduction}

Optimization problems appear in all parts of our lives. These problems are classified according to the type of objective functions. For example, when the objective function is a set-valued function/mapping/map, the optimization problem is named set-valued optimization problem (shortly, SVOP). Recently, SVOP has attracted increasing attention because it has many applications such as finance, control theory, game theory, engineering, statistic, etc.

In the SVOP, there are several approaches to solve these optimization problems. Vector and set approaches are the most commonly used types. The first used is the vector approach. In this approach, efficient vectors of the image set of the objective map are investigated. In order to be a solution of a point, the image set of this point has to contain an efficient vector of the image set. The set approach, which is given by Kuroiwa [16,17], depends on the comparison among values of the objective map. So, an order relation must be used to compare sets in this approach. More information about these approaches and the solution concepts are also available in $[4,6,8-15,17,18]$, and references therein. In this current investigation, vector and set approaches are considered.

Firstly, Kuroiwa et al. [19] mentioned about set relation based on the ordering cone. Then, they defined six order relations. They gave relationships with each other. By using these order relations, the set optimization approach is constructed by Kuroiwa [16,17]. Kuroiwa obtained the solutions of SVOP with respect to (shortly, wrt) set approach. Jahn \& Ha [6] obtained some new order relations for SVOP. Two new partial order relations are defined by Karaman et al. [13] for SVOP. There are still sets that can not be compared with these partial and the other order relations. That's why we define a new order relation to compare such sets in this paper.

In order to solve $S V O P$, some methods are used as vectorization, scalarization, directional derivative, subdifferential, embedding space, and so on [1-4,7-9,11-15,21,23]. The well-known scalarization functions are Gerstewitz, the oriented function of Hiriart-Urruty [5] and generalizations of them. Hernández \& Rodríguez-Marín [4] found some optimality conditions for SVOP via derived an extension of Gerstewitz function. Recently, some authors like Khushboo \& Lalitha [15], Xu \& Li [23], Jiménez et al. [7], Ansari et al. [1] and Chen et al. [2] obtained scalarizations via some extension of the oriented function. 
A new relation on the subset of the space is defined via $\ell$-difference in this work. We show that this order relation is a pre-order on the family of nonempty sets. Some properties of this pre-order relation are obtained. This pre-order relation is compared with some well-known order relations in the literature. Also, the solutions of SVOP wrt set and vector approaches are examined.

The layout of the study is ordered as follows: The basic definitions and concepts of SVOP are stated and mentioned in section 2. In section 3, a pre-order relation is introduced and some properties are discovered. In section 4, after the solutions concept of SVOP are recalled wrt set approach, the solutions of SVOP are compared according to set and vector approaches.

\section{Mathematical Preliminaries}

In this study, $Y$ is denoted as a normed space and $X$ is a vector space. Let $K \subset Y$ be given. If $\lambda x \in K$ for all $x \in K, \lambda>0$, then $K$ is called a cone. Assume that cone $K$ is a convex, pointed $\left(K \cap(-K)=\left\{0_{Y}\right\}\right)$ and closed with the nonempty interior, and $Y$ be ordered by cone $K . \mathscr{P}(Y)$ is denoted the family of proper and nonempty subsets of $Y$, that is, $\mathscr{P}(Y):=\{A \subset Y$ : $A \neq Y$ and $A$ is nonempty $\}$. Topological interior and convex hull of any set $A \in \mathscr{P}(Y)$ are indicated by int $(A)$ and $\operatorname{conv} A$, respectively.

It is denoted that the algebraic sum of $A$ and $B$ by $A+B$, the algebraic difference of $A$ and $B$ by $A-B$, Minkowski (Pontryagin) difference of $A$ and $B$ by $A \dot{-} B:=\{x \in Y \mid x+B \subset A\}$ and $\ell$-difference of $A$ and $B$ by $A \ominus_{\ell} B:=\{x \in Y \mid x+B \subset$ $A+K\}=(A+K) \dot{-} B$ for any $A, B \in \mathscr{P}(Y)$. Readers can find more information about these in [20-22].

The cone $K$ induces an ordering relations on $Y$ as follow: For $x, x^{\prime} \in Y, x \leq_{K} x^{\prime}$ iff $x^{\prime}-x \in K$, and $x<_{K} x^{\prime}$ iff $x^{\prime}-x \in i n t(K)$.

Let $A \in \mathscr{P}(Y)$ be a set and $a_{0} \in A$. If $A \cap\left(a_{0}-K\right)=\left\{a_{0}\right\}\left(A \cap\left(a_{0}+K\right)=\left\{a_{0}\right\}\right)$, then $a_{0}$ is called a minimal (maximal) point of $A$. The set of all minimal and maximal points of $A$ is indicated by $\min A$ and $\max A$, respectively. Likewise, if $A \cap\left(a_{0}-\operatorname{int}(K)\right)=\emptyset\left(A \cap\left(a_{0}+\operatorname{int}(K)\right)=\emptyset\right)$, then we say that $a_{0}$ is a weak minimal (weak maximal) point of $A$.

Note that the binary relation $\preceq$ on $\mathscr{S} \subset \mathscr{P}(Y)$ is called a pre-order on $\mathscr{S}$ if $\preceq$ is reflexive and transitive. Also, if pre-order relation $\preceq$ is antisymmetric then the order relation is called a partial order on $\mathscr{S}$.

Definition 2.1. Let $\mathscr{S} \subset \mathscr{P}(Y)$ and $A, B, C \in \mathscr{S}$ be any sets. The relation $\preceq$ on $\mathscr{S}$ is said to be

(i) compatible with the addition if $A \preceq B$ implies $A+C \preceq B+C$,

(ii) compatible with positive scalar multiplication if $A \preceq B$ implies $\lambda A \preceq \lambda B$ for all scalars $\lambda>0$.

Let $F: X \rightrightarrows Y$ be a set-valued function such that $F(x) \in \mathscr{P}(Y)$ for all $x \in X$, and $\operatorname{dom}(F):=\{x \in X \mid F(x) \neq \emptyset\}$ be efficient domain set of the set-valued mapping $F . S \subset \operatorname{dom}(F)$ be given. Basic $S V O P$ is described by

$$
\text { SVOP }\left\{\begin{array}{l}
\min (\max ) F(x) \\
\text { s.t. } x \in S .
\end{array}\right.
$$

We denote the problem by $(v-S V O P)$ when $S V O P$ considers wrt vector approach. Efficient points of the set $F(S):=$ $\bigcup_{x \in S} F(x)$ are investigated to solve $(v-S V O P)$, that is, if $F\left(x_{0}\right)$ contains a minimal (maximal) point of $F(S)$, then $x_{0} \in S$ is called a solution of $(v-S V O P)$. In the same way, if $F\left(x_{0}\right)$ contains a weak minimal (weak maximal) point of $F(S)$, then $x_{0} \in S$ is entitled a weak solution of $(v-S V O P)$.

In the set approach, a comparison among the values of the set-valued mapping is considered. Namely, efficient sets of $\mathscr{F}(S):=\{F(x) \mid x \in S\}$ are investigated to solve $S V O P$. So, an order relation is needed to solve a SVOP by using the set approach. In the following definition, some order relations are given:

Definition 2.2. $[6,13,17]$ Let $A, B \in \mathscr{P}(Y)$.

(i) $A \preceq^{1} B$ is described by $\forall a \in A, \forall b \in B, a \leq_{K} b$,

(ii) $A \preceq^{2} B$ is described by $\exists a \in A$ such $\forall b \in B, a \leq_{K} b$,

(iii) $A \preceq^{3} B$ is described by $\forall b \in B, \exists a \in A$ such $a \leq_{K} b$,

(iv) $A \preceq^{4} B$ is described by $\exists b \in B$, such $\forall a \in A, a \leq_{K} b$,

(v) $A \preceq^{5} B$ is described by $\forall a \in A, \exists b \in B$ such that $a \leq_{K} b$,

(vi) $A \preceq^{6} B$ is described by $\exists a \in A, \exists b \in B$ such that $a \leq_{K} b$,

(vii) $A \preceq^{s} B$ is described by $A \preceq^{3} B$ and $A \preceq^{5} B$, 
(viii) $A \preceq^{m_{1}} B$ is described by $(B \dot{-} A) \cap K \neq \emptyset$,

(ix) $A \preceq^{m_{2}} B$ is described by $(A \dot{-} B) \cap(-K) \neq \emptyset$.

It is assumed that $* \in\left\{1,2,3,4,5,6, s, m_{1}, m_{2}\right\}$ in the rest of the study. In the set approach, the problem is denoted by $(*-S V O P)$ when $S V O P$ considers wrt order relation $\preceq *$. The efficient set of $\mathscr{F}(S)$ is investigated to solve $(*-S V O P)$. That is, if $F\left(x_{0}\right) \in \mathscr{F}(S)$ is a minimal (resp., maximal) set of $\mathscr{F}(S)$, then $x_{0}$ is called a solution of $(*-S V O P)$. Similarly, if $F\left(x_{0}\right) \in \mathscr{F}(S)$ is a weak minimal (resp., weak maximal) set of $\mathscr{F}(S)$, then $x_{0}$ is named a weak solution of $(*-S V O P)$.

\section{A new Order Relation for Set Approach}

In this section, a pre-order relation is derived by using $\ell$-difference and some properties of this relation are examined.

Definition 3.1. Let $A, B \in \mathscr{P}(Y)$. $\ell_{1}$ relation is defined as

$$
A \preceq^{\ell_{1}} B: \Longleftrightarrow\left(B \ominus_{\ell} A\right) \cap K \neq \emptyset .
$$

When $A$ and $B$ are taken as singleton, there is a relation between $\preceq^{\ell_{1}}$ and vector order relation $\leq_{K}$ on $Y$ as:

$$
a \leq_{K} b \Longrightarrow\{a\} \preceq^{\ell_{1}}\{b\}
$$

for any $A=\{a\}, B=\{b\}$ and $a, b \in Y$.

When two sets don't compare wrt partial order relation $\preceq^{m_{1}}$, they may be compared wrt order relation $\preceq^{\ell_{1}}$. For example, when $A=\left\{(x, 0) \in \mathbb{R}^{2} \mid 1 \leq x \leq 3\right\}$ and $B=\left\{(0, y) \in \mathbb{R}^{2} \mid 1 \leq y \leq 3\right\}$, we have $A \preceq \ell^{\ell_{1}} B$ and $A \swarrow^{m_{1}} B$.

Now, some properties of $\preceq^{\ell_{1}}$ are presented.

Proposition 3.2. The order relation $\preceq^{\ell_{1}}$ has the following properties;

(i) $\preceq^{\ell_{1}}$ is compatible with the addition,

(ii) $\preceq^{\ell_{1}}$ is compatible with the positive scalar multiplication.

Proof.

(i) Let $A, B, C \in \mathscr{P}(Y)$ and $A \preceq^{\ell_{1}} B$ be given. Since $A \preceq^{\ell_{1}} B$, we have $\left(B \ominus_{\ell} A\right) \cap K \neq \emptyset$. That means there exists $\bar{x} \in K$ such that $\bar{x} \in B \ominus_{\ell} A$. Then, we get $\bar{x}+A \subset B+K$. So, $\bar{x}+A+C \subset B+C+K$, that is $\bar{x} \in(B+C) \ominus_{\ell}(A+C)$. Therefore,

$$
\left[(B+C) \ominus_{\ell}(A+C)\right] \cap K \neq \emptyset .
$$

Thus, we obtain $A+C \preceq^{\ell_{1}} B+C$ that implies $\preceq^{\ell_{1}}$ is compatible with the addition.

(ii) Let $A \preceq{ }^{\ell_{1}} B$. We show that $\lambda A \preceq{ }^{\ell_{1}} \lambda B$ for all scalars $\lambda>0$. Since $A \preceq{ }^{\ell_{1}} B$, there exists an $\bar{x} \in K$ such $\bar{x} \in B \ominus_{\ell} A$, i.e., $\bar{x}+A \subset B+K$. So, we have $\lambda \bar{x}+\lambda A \subset \lambda B+\lambda K=\lambda B+K$ and $\lambda \bar{x} \in K$ because $K$ is cone. Then, we obtain $\left(\lambda B \ominus_{\ell} \lambda A\right) \cap K \neq \emptyset$, i.e., $\lambda A \preceq{ }^{\ell_{1}} \lambda B$. Hence, $\preceq^{\ell_{1}}$ is compatible with the positive scalar multiplication.

Proposition 3.3. The order relation $\preceq^{\ell_{1}}$ has the following properties;

(i) $\preceq^{\ell_{1}}$ is reflexive,

(ii) $\varliminf^{\ell_{1}}$ is transitive.

Proof.

(i) Let $A \in \mathscr{P}(Y)$. Because $0_{Y} \in A \ominus_{\ell} A$ and $0_{Y} \in K$, we have $\left(A \ominus_{\ell} A\right) \cap K \neq \emptyset$. Hence, $A \preceq^{\ell_{1}} A$.

(ii) Assume that $A \preceq{ }^{\ell_{1}} B$ and $B \preceq^{\ell_{1}} C$ for any $A, B, C \in \mathscr{P}(Y)$. We have $\left(B \ominus_{\ell} A\right) \cap K \neq \emptyset$ since $A \preceq{ }^{\ell_{1}} B$. Then, there exists $x_{1} \in K$ such

$$
x_{1}+A \subset B+K \text {. }
$$

Since $B \preceq{ }^{\ell_{1}} C,\left(C \ominus_{\ell} B\right) \cap K \neq \emptyset$ yields. Then, there exists $x_{2} \in K$ such

$$
x_{2}+B \subset C+K \text {. }
$$

From (3.1) and (3.2) we get $x_{1}+x_{2}+A \subset x_{2}+B+K \subset C+K+K=C+K$. As $x_{1}+x_{2}+A \subset C+K$ and $x_{1}+x_{2} \in K$, we obtain $\left(C \ominus_{\ell} A\right) \cap K \neq \emptyset$, i.e., $A \preceq{ }^{\ell_{1}} C$. 
Remark 3.4. The order relation $\preceq^{\ell_{1}}$ isn't antisymmetric. For example, let $Y=\mathbb{R}^{2}, K=\mathbb{R}_{+}^{2}, A=\{(1,1)\}$ and $B=\{(2,2)\}$ be given. Then, we get $B \ominus_{\ell} A=\left\{x \in \mathbb{R}^{2} \mid x+A \subset B+K\right\}=[1, \infty) \times[1, \infty)$ and $A \ominus_{\ell} B=\left\{x \in \mathbb{R}^{2} \mid x+B \subset A+K\right\}=$ $[-1, \infty) \times[-1, \infty)$. So, we have $\left(B \ominus_{\ell} A\right) \cap K \neq \emptyset$ and $\left(A \ominus_{\ell} B\right) \cap K \neq \emptyset$, i.e., $A \preceq^{\ell_{1}} B$ and $B \preceq^{\ell_{1}} A$. But $A \neq B$. Hence, $\preceq^{\ell_{1}}$ isn't antisymmetric.

Corollary 3.5. The order relation $\preceq^{\ell_{1}}$ is a pre-order relation on $\mathscr{P}(Y)$.

Now, a relation between the order relation $\preceq^{\ell_{1}}$ and order relation $\preceq^{m_{1}}$ are given.

Proposition 3.6. Let $A, B \in \mathscr{P}(Y)$. If $A \preceq^{m_{1}} B$, then $A \preceq^{\ell_{1}} B$.

Proof. Let $A \preceq^{m_{1}} B$, i.e., $(B \dot{-} A) \cap K \neq \emptyset$ be given. There exists an $x \in K$ such $x+A \subset B$. Because $K$ is pointed, we get $x+A \subset B+K$, i.e., $x \in B \ominus_{\ell} A$. As $x \in K$ and $\left(B \ominus_{\ell} A\right) \cap K \neq \emptyset$, we obtain $A \preceq^{\ell_{1}} B$

Note that $\preceq^{\ell_{1}}$ doesn't imply $\preceq^{m_{1}}$. This is presented in the following example.

Example 3.7. Let $Y=\mathbb{R}^{2}, K=\mathbb{R}_{+}^{2}, A=\operatorname{conv}\{(0,0),(1,2)\}$ and $B=\operatorname{conv}\{(0,0),(2,1)\}$.
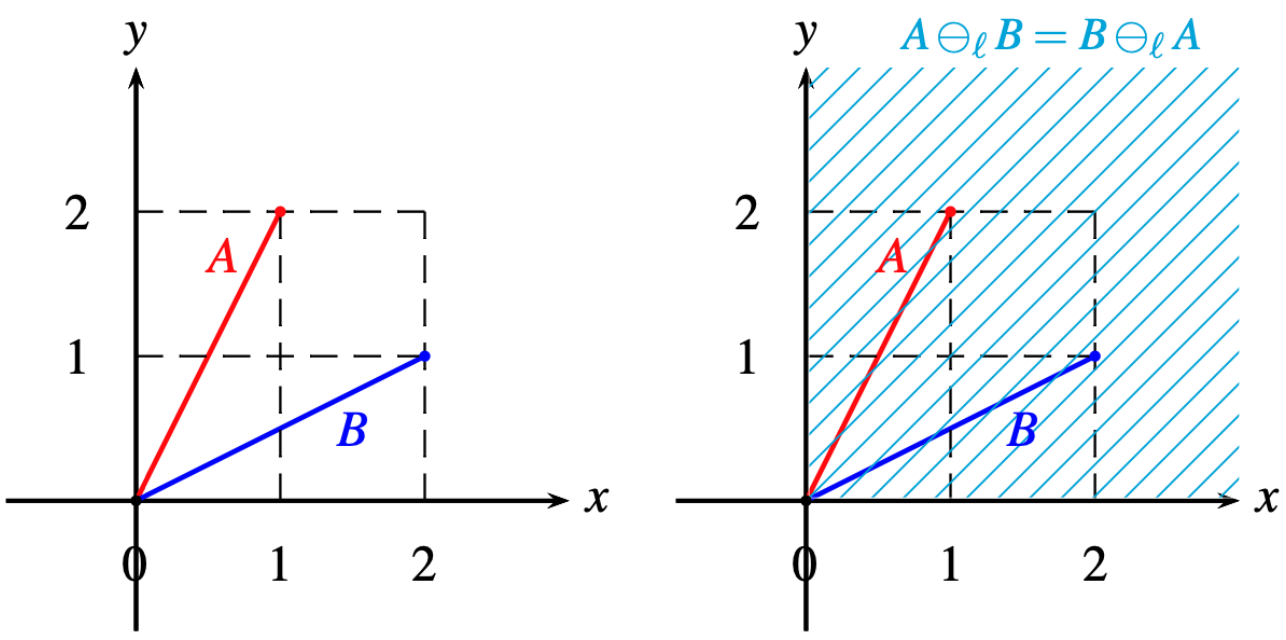

Figure 3.1. $A=\operatorname{conv}\{(0,0),(1,2)\}, B=\operatorname{conv}\{(0,0),(2,1)\}, A \ominus_{\ell} B$ and $B \ominus_{\ell} A$

As seen in Figure 3.1, since $\left(B \ominus_{\ell} A\right)=K$, we have $\left(B \ominus_{\ell} A\right) \cap K=K$, i.e., $A \preceq^{\ell_{1}} B$. On the other hand, as $B \dot{-} A=\emptyset$, we get $(B \dot{-} A) \cap K=\emptyset$, i.e., $A \swarrow^{m_{1}} B$.

In the following definition, strict version of $\preceq^{\ell_{1}}$ is given.

Definition 3.8. Let $A, B \in \mathscr{P}(Y)$. The strict $\ell_{1}$ order relation is defined by

$$
A \prec^{\ell_{1}} B: \Longleftrightarrow\left(B \ominus_{\ell} A\right) \cap \operatorname{int}(K) \neq \emptyset
$$

Note that $\prec^{\ell_{1}}$ implies $\preceq^{\ell_{1}}$. Namely, if $A \prec^{\ell_{1}} B$, then $A \preceq^{\ell_{1}} B$ for all $A, B \in \mathscr{P}(Y)$.

Remark 3.9. The order relation $\prec^{\ell_{1}}$ is compatible with not only the addition but also the positive scalar multiplication. Moreover, it is reflexive and transitive. But it isn't antisymmetric.

One of the most important problems in the set order relations is that some sets can not be compared according to any order relation. Although two sets may not be compared wrt order relation $\preceq^{*}$, these sets can be compared wrt $\ell_{1}$ order relation. This is illustrated in the accompanying example.

Example 3.10. Let $K=\mathbb{R}_{+}^{2}, A=\left\{(x, y) \in \mathbb{R}^{2} \mid 1 \leq x \leq 2\right.$ and $\left.3 \leq y \leq 4\right\}$ and $B=\left\{(x, y) \in \mathbb{R}^{2} \mid 3 \leq x \leq 4\right.$ and $\left.1 \leq y \leq 2\right\}$. As seen Figure 3.2, while $A \swarrow^{*} B$ we obtain $\left(B \ominus_{\ell} A\right) \cap K \neq \emptyset$, i.e., $A \preceq^{\ell_{1}} B$. 

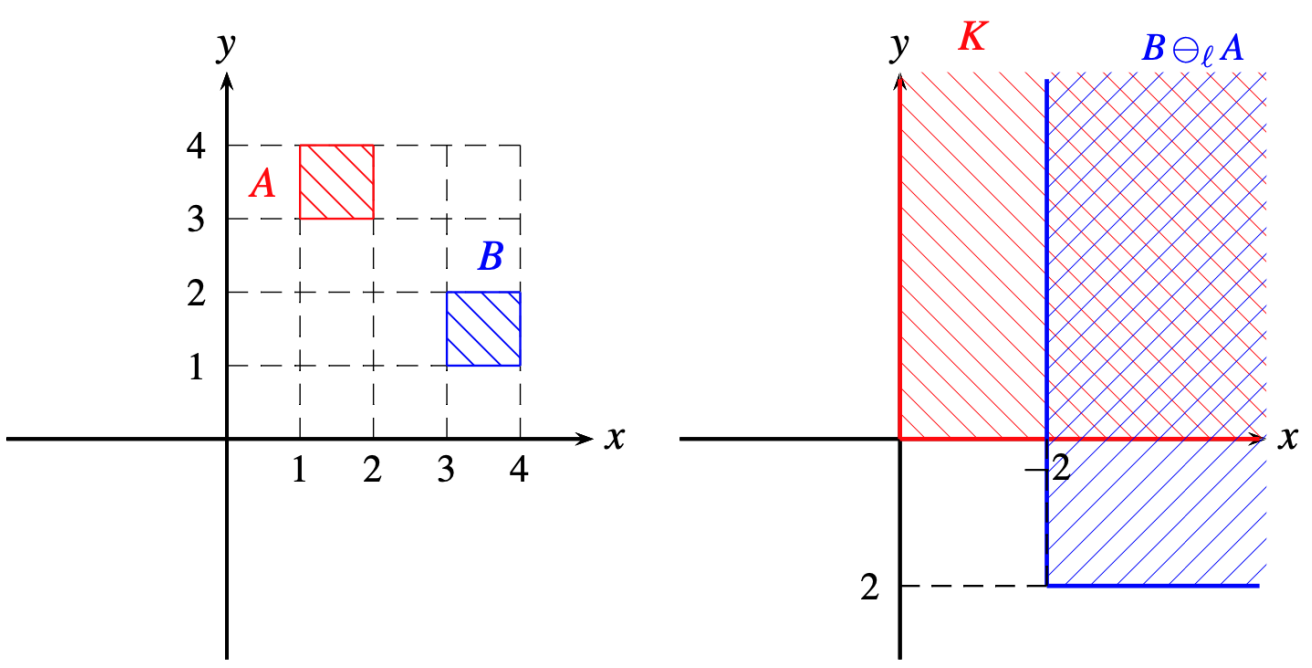

Figure 3.2. $A=\left\{(x, y) \in \mathbb{R}^{2} \mid 1 \leq x \leq 2\right.$ and $\left.3 \leq y \leq 4\right\}, B=\left\{(x, y) \in \mathbb{R}^{2} \mid 3 \leq x \leq 4\right.$ and $\left.1 \leq y \leq 2\right\}$ and $B \ominus_{\ell} A$

Proposition 3.11. Let $A, B \in \mathscr{P}(Y)$. Then, the following assertions are satisfied:

(i) If there exist $a \in A$ and $b \in B$ such that $b \leq_{K}$ a, then $A \preceq{ }^{\ell_{1}} B$,

(ii) if there exist $a \in A$ and $b \in B$ such that $b<_{K}$ a, then $A \prec^{\ell_{1}} B$.

Proof. (i) Assume that there exist $a \in A$ and $b \in B$ such that $b \leq_{K} a$. By contradiction, suppose that $A \swarrow^{l_{1}} B$. Then, $\left(B \ominus_{\ell} A\right) \cap K=\emptyset$, and we have $k+A \not \subset B+K$ for all $k \in K$. So, $k+a \notin B+K$ for all $k \in K, a \in A$. From here, we get $k+a \notin b+K$ for all $k \in K, a \in A$ and $b \in B$. Let $k=0_{Y}$, then $a \notin b+K$ for all $a \in A$ and $b \in B$. Therefore, for all $a \in A$ and $b \in B$ we get $b \not_{K} a$, which is a contradict.

(ii) This can be proven by similarly to (i).

\section{Solution Concepts of SVOP According to Set and Vector Approaches}

In this part of the study, we obtain relations between the solutions of $(v-S V O P)$ and $\left(\ell_{1}-S V O P\right)$.

In the following definition, the efficient elements of a family are given wrt pre-order relation $\preceq^{\ell_{1}}$.

Definition 4.1. Let $\mathscr{S} \subset \mathscr{P}(Y)$ and $A \in \mathscr{S}$ be given. We call that

(i) A is an $\ell_{1}$-minimal ( $\ell_{1}$-maximal) element of $\mathscr{S}$ iff

$$
B \preceq^{\ell_{1}} A \text { for some } B \in \mathscr{S} \Longrightarrow A \preceq^{\ell_{1}} B \quad\left(A \preceq^{\ell_{1}} B \text { for some } B \in \mathscr{S} \Longrightarrow B \preceq^{\ell_{1}} A\right),
$$

(ii) $A$ is a weak $\ell_{1}$-minimal (weak $\ell_{1}$-maximal) element of $\mathscr{S}$ iff

$$
B \prec^{\ell_{1}} A \text { for some } B \in \mathscr{S} \Longrightarrow A \prec^{\ell_{1}} B \quad\left(A \prec^{\ell_{1}} B \text { for some } B \in \mathscr{S} \Longrightarrow B \prec^{\ell_{1}} A\right) \text {. }
$$

If we consider $S V O P$ wrt $\ell_{1}$ order relation, then problem is denoted by

$$
\left(\ell_{1}-S V O P\right)\left\{\begin{array}{l}
\min (\max ) F(x) \\
\text { s.t. } x \in S .
\end{array}\right.
$$

Let $x_{0} \in S$ be given. $x_{0}$ is called a solution of $\left(\ell_{1}-S V O P\right)$ if $F\left(x_{0}\right) \in \mathscr{F}(S)$ is an $\ell_{1}$-minimal $\left(\ell_{1}\right.$-maximal) set of $\mathscr{F}(S)$. Similarly, $x_{0}$ is called a weak solution of $\left(\ell_{1}-S V O P\right)$ if $F\left(x_{0}\right) \in \mathscr{F}(S)$ is a weak $\ell_{1}$-minimal (weak $\ell_{1}$-maximal) set of $\mathscr{F}(S)$.

The solution of $\left(\ell_{1}-S V O P\right)$ may not be the solution of $(v-S V O P)$. Now, we will give an example related to this situation. 


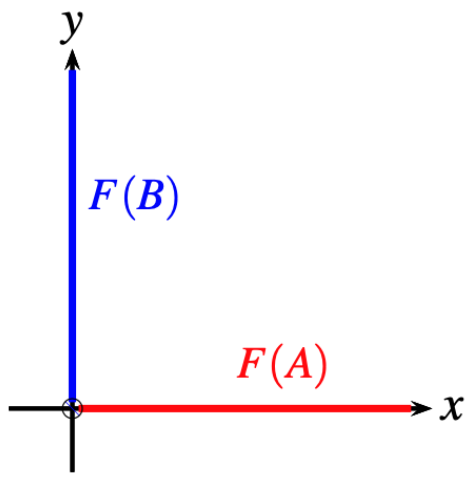

Figure 4.1. $F(A)=\left\{(x, 0) \in \mathbb{R}^{2} \mid x>0\right\}$ and $F(B)=\left\{(0, y) \in \mathbb{R}^{2} \mid y>0\right\}$

Example 4.2. Let $Y=\mathbb{R}^{2}, K=\mathbb{R}_{+}^{2}$, set-valued map $F:\{A, B\} \rightrightarrows \mathbb{R}^{2}$ be defined as $F(A)=\left\{(x, 0) \in \mathbb{R}^{2} \mid x>0\right\}$ and $F(B)=\left\{(0, y) \in \mathbb{R}^{2} \mid y>0\right\}$. Consider the following set-valued optimization problem

$$
\text { SVOP }\left\{\begin{array}{l}
\min F(x) \\
\text { s.t. } x \in\{A, B\} .
\end{array}\right.
$$

As seen in Figure 4.1, $F(A) \cap \min \{F(A) \cup F(B)\}=\emptyset$ and $F(B) \cap \min \{F(A) \cup F(B)\}=\emptyset$. So, $A$ and $B$ pairs of sets are not a solution of $(v-S V O P)$. On the other hand, $A$ and $B$ are solution of $\left(\ell_{1}-S V O P\right)$ because $F(A) \preceq^{\ell_{1}} F(B)$ implies $F(B) \preceq^{\ell_{1}} F(A)$, and $F(B) \preceq^{\ell_{1}} F(A)$ implies $F(A) \preceq^{\ell_{1}} F(B)$.

Conversely, the solution of $(v-S V O P)$ may not be the solution of $\left(\ell_{1}-S V O P\right)$. The following example is related to this situation.

Example 4.3. Let $Y=\mathbb{R}^{2}, K=\mathbb{R}_{+}^{2}$, set-valued map $F:\{1,2\} \rightrightarrows \mathbb{R}^{2}$ be defined as $F(1)=\left\{(x, y) \in \mathbb{R}^{2} \mid x=y\right.$ and $\left.x \geq 0\right\}$ and $F(2)=\left\{(x, y) \in \mathbb{R}^{2} \mid y=-x\right.$ and $\left.x \geq 0\right\}$. Let's consider the following problem

$$
\text { SVOP }\left\{\begin{array}{l}
\min F(x) \\
\text { s.t. } x \in\{1,2\} .
\end{array}\right.
$$

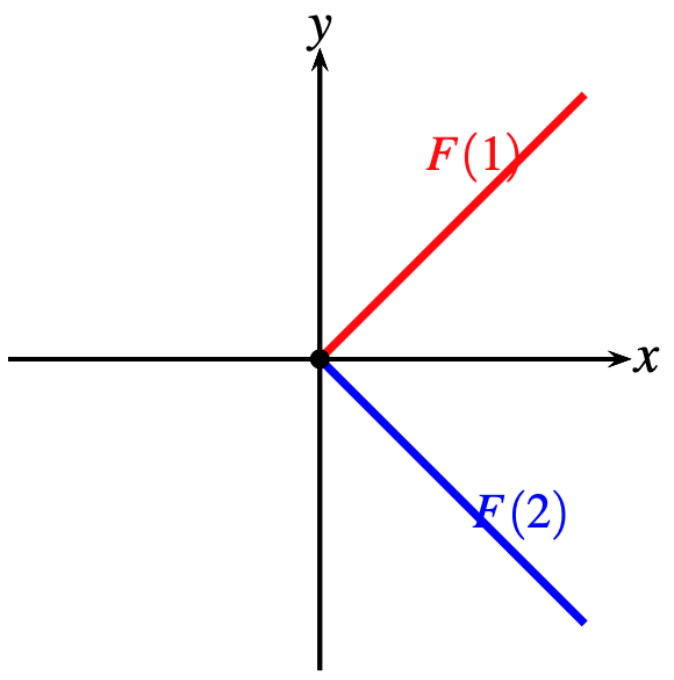

Figure 4.2. $F(1)=\left\{(x, y) \in \mathbb{R}^{2} \mid x=y\right.$ and $\left.x \geq 0\right\}$ and $F(2)=\left\{(x, y) \in \mathbb{R}^{2} \mid y=-x\right.$ and $\left.x \geq 0\right\}$

As seen in Figure 4.2, because $\min \{F(1) \cup F(2)\} \cap F(1) \neq \emptyset$ and $\min \{F(1) \cup F(2)\} \cap F(2) \neq \emptyset, 1$ and 2 are solution of $(v-S V O P)$. 
Since $\left(F(2) \ominus_{\ell} F(1)\right) \cap K \neq \emptyset$, we have $F(1) \preceq^{\ell_{1}} F(2)$. As $F(1) \ominus_{\ell} F(2)=\emptyset$, we obtain $\left(F(1) \ominus_{\ell} F(2)\right) \cap K=\emptyset$. Hence, we get $F(2) \npreceq^{\ell_{1}} F(1)$. Because $F(1) \preceq{ }^{\ell_{1}} F(2)$ doesn't imply $F(2) \preceq^{\ell_{1}} F(1), 2$ isn't a solution of $\left(\ell_{1}-S V O P\right)$. Although 2 is a solution of $(v-S V O P)$, it isn't a solution of $\left(\ell_{1}-S V O P\right)$.

\section{Conclusion}

In this study, a new pre-order relation on the family of nonempty sets is introduced, and set-valued optimization problems $\left(\ell_{1}-S V O P\right)$ are derived. Some optimality conditions can be obtained by using different tools such as vectorization, directional derivative, scalarization, subdifferential etc. for $\left(\ell_{1}-S V O P\right)$.

\section{References}

[1] Q. H. Ansari, E. Köbis, P. K. Sharma, Characterizations of multiobjective robustness via oriented distance function and image space analysis, J. Optim. Theory. Appl., 181(3) (2019), 817-839.

[2] J. Chen, Q. H. Ansari, J.-C. Yao, Characterizations of set order relations and constrained set optimization problems via oriented distance function, Optimization, 66(11) (2017), 1741-1754.

[3] G. P. Crespi, I. Ginchev, M. Rocca, First-order optimality conditions in set-valued optimization, Math. Meth. Oper. Res., 63(1) (2006), 87-106.

[4] E. Hernández, L. Rodríguez-Marín, Nonconvex scalarization in set optimization with set-valued maps, J. Math. Anal. Appl., 325(1) (2007), 1-18.

[5] J. B. Hiriart-Urruty, Tangent cone, generalized gradients and mathematical programming in Banach spaces, Math. Oper. Res., 4(1) (1979), 1-97.

[6] J. Jahn, T. X. D. Ha, New order relations in set optimization, J. Optimiz. Theory. App., 148(2) (2011), $209-236$.

[7] B. Jiménez, V. Novo, A. Vílchez, Characterization of set relations through extensions of the oriented distance, Math. Method. Oper. Res., 91 (2020), 89-115.

[8] A. A. Khan, C. Tammer, C. Zălinescu, Set-valued Optimization: An Introduction with Applications, Springer-Verlag, Berlin, 2015.

[9] E. Karaman, Gömme fonksiyonu kullanılarak küme optimizasyonuna göre verilen küme değerli optimizasyon problemlerinin optimallik koşulları, Süleyman Demirel Üniversitesi Fen Edebiyat Fakültesi Fen Dergisi, 14 (2019), $105-111$.

[10] E. Karaman, Nonsmooth set variational inequality problems and optimality criteria for set optimization, Miskolc. Math. Notes., 21(1) (2020), 229-240.

[11] E. Karaman, M. Soyertem, İ.Atasever Güvenç, Optimality conditions in set-valued optimization problem with respect to a partial order relation via directional derivative, Taiwan. J. Math., 24(3) (2020), 709-722.

[12] E. Karaman, İ. Atasever Güvenç, M. Soyertem, D.Tozkan, M. Küçük, Y. Küçük, A vectorization for nonconvex set-valued optimization, Turk. J. Math., 42 (2018), 1815-1832.

[13] E. Karaman, M. Soyertem, İ. Atasever Güvenç, D. Tozkan, M. Küçük, Y. Küçük, Partial order relations on family of sets and scalarizations for set optimization, Positivity, 22(3) (2018), 3783-802.

[14] E. Karaman, İ. Atasever Güvenç, M. Soyertem, Optimality conditions in set-valued optimization problems with respect to a partial order relation by using subdifferentials, Optimization, 70(3) (2021) 613-630.

[15] Khushboo, C. S. Lalitha, Scalarizations for a set optimization problem using generalized oriented distance function, Positivity, 23(5) (2019), 1195-1213.

[16] D. Kuroiwa, The natural criteria in set-valued optimization, RIMS Kokyuroku, 1031(2) (1998), 85-90.

[17] D. Kuroiwa, On set-valued optimization, Nonlinear. Anal-Theor., 47(2) (2001), 1395-1400.

[18] D. Kuroiwa, Existence theorems of set optimization with set-valued maps, J. Inf. Optim. Sci., 24(1) (2003), 73-84.

[19] D. Kuroiwa, T. Tanaka, T. X. D. Ha, On cone convexity of set-valued maps, Nonlinear. Anal-Theor., 30(3) (1997), 1487-1496.

[20] D. Pallaschke, R. Urbański, Pairs of Compact Convex Sets, Kluwer Academic Publishers, Dordrecht, (2002).

[21] M. Pilecka, Optimality conditions in set-valued programming using the set criterion, Thecnical University of Freiberg, 2014 (2014). 
[22] R. Schneider, Convex Bodies: The Brunn-Minkowski Theory, Cambridge University Press, Cambridge, 1993.

[23] Y. D. Xu, S. J. Li, A new nonlinear scalarization function and applications, Optimization, 65(1) (2016), $207-231$. 